\title{
Electric Field Recovery after Lightning Flash and Regeneration of Charge in Thundercloud
}

\author{
By Minoru Nakano \\ The Research Institute of Atmospherics, Nagoya University, Toyokawa, Aichi 442 \\ (Manuscript received 26 August 1974, in revised form 8 April 1975)
}

\begin{abstract}
The relaxation time of exponential recovery of the electric field after lightning flash occurring at the distance beyond $10 \mathrm{~km}$ is discussed. The relaxation time decreases with increasing distance, but the values obtained in the present study are found to be about 1.5 to 2 times larger than the values obtained by other workers. For close flashes, the field recoveries show almost linear change with time, which is apparently different from that for distant flashes. It is found that there is a good correlation between the average recovery rate and the occurence frequency of flashes for two storms and that the field recovery rate positively correlates with the regeneration rate of charges in thunderclouds. Hence, it is possible to estimate the regeneration rate of charges in thunderclouds from the counting of the occurrence frequency of flashes for any flash distance. The regeneration rate after lightning flash is found to be about $1-10 \mathrm{coul} / \mathrm{sec}$ for the two storms.
\end{abstract}

\section{Introduction}

The time variation of electric field measured on the ground after a lightning flash is often called the field recovery curve and shows usually exponential decrease for a distant flash. The characteristics of recovery curves were investigated by many workers. Smith (1958) showed that the relaxation time of exponential recovery for a distant flash depends on the intensity of the electric field change, that is, on the distance to a flash. Malan (1967), Michnowski (1969) and Illingworth (1971) reported similar dependency on the distance. An experimental formula for the relationship between relaxation time and distance was given by Illingworth (1971). However, their results did not agree with each other. This would suggest that the electric conductivity of the air surrounding thunderclouds deviates from the value produced by the ionization by cosmic rays.

It was first pointed out by Tamura (1954) that the exponential recovery was due not only to the charges in clouds but to the space charges in the surrounding atmosphere. Although Tamura gave an analytical solution of the equations for the electric field and the charge conservation, he could not explain the dependency of relaxation time on the distance to a flash. Recently, Illingworth
(1972) obtained another solution of numerical computation without taking into account the effect by point discharge current. While he did not refer to the differences from other investigations, the solution was in good agreement with his observations under the assumed conductivity profile indicating exponential increase with height. Hence, it is evident that more data are needed to discuss this point.

For a close flash, the recovery curves are nearly linear rather than exponential and often involve irregular changes. Although Smith (1958) suggests that the linear recovery is caused by rainfall at the observation site, this linear recovery may be correlated directly with the electrical activity in clouds, and the recovery curves for close flashes may be expected to comprise useful informations for regeneration or separation of charges in thunderclouds.

In this paper, first, the relaxation times of exponential field recoveries for medium-distant and distant flashes are investigated comparing with those by other investigators. Secondly, the influence of rainfall at the observation site on the field recovery for the close flash will be discussed. Finally, the relationship between the field recovery rate for close flashes and the occurrence frequency of flashes are shown, and the regeneration rate 
of charges after flashes will be discussed. Here, the flash which occurrs in a range between $10 \mathrm{~km}$ and $20 \mathrm{~km}$ is defined as a medium-distant flash, the flash beyond $20 \mathrm{~km}$ a distant flash and the flash less than $10 \mathrm{~km}$ a close flash.

\section{Measurements}

The electric field was measured with a field-mill, which was calibrated by a vertical antenna with an ionizer (Americium) on its top. The time resolution of the field-mill is about $0.4 \mathrm{sec}$, but that of the whole measuring system is about $1 \mathrm{sec}$ which is mainly controlled by a recorder. The electric field change measured with a vertical antenna and the thunder were recorded simultaneously on a magnetic data recorder in order to identify whether or not a flash is cloud-to-ground discharge and to determine the approximate distance to the flash. A rainfall region was detected with a 3-cm PPI radar and rainfall rate was measured by a rain guage with a sensitivity of $0.5 \mathrm{~mm}$. The distance from the observation site to a flash was determined roughly from the time difference between occurrence of a field change and arrival of thunder. When this method could not be used, flashes were assumed to locate in a rainfall region detected by a radar. The data were obtained in the summer of 1973 at Imaiti, Totigi, Japan.

\section{Medium-distant and distant flash}

Typical recovery curves are shown in Figs. 1 and 2, where the data of distant flashes and close flashes are shown respectively. It is noticed that the shapes of recovery curves in the two figures are quite different, though the occurence frequencies of flashes are almost equal in the two figures. Recovery curves are usually exponential for distant flashes, and the change of occurrence rate of the exponential field recovery with the distance is shown in Fig. 3. The numbers of flashes for each storm are shown inside the parentheses in this figure. Fig. 3 shows that the occurrence rate does not depend on the number of flashes, but depend on the distance, and it increase with distance.

The variation of relaxation time with distance has also been examined, and the result is shown in Fig. 4. Here, the electric field intensity, $E(t)$, for the exponential field recovery is expresses as follows

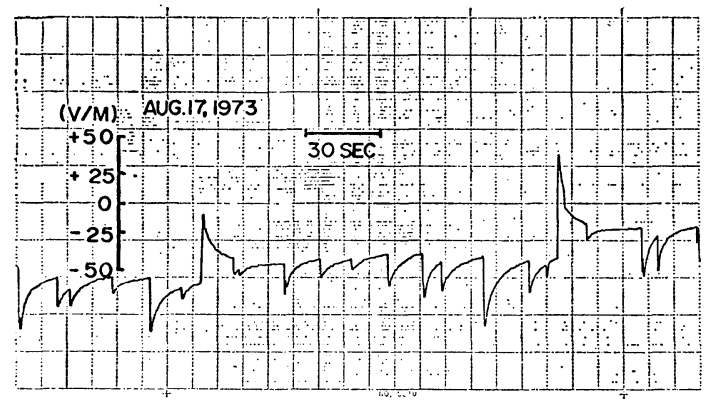

Fig. 1. Examples of field recoveries after distant flashes.

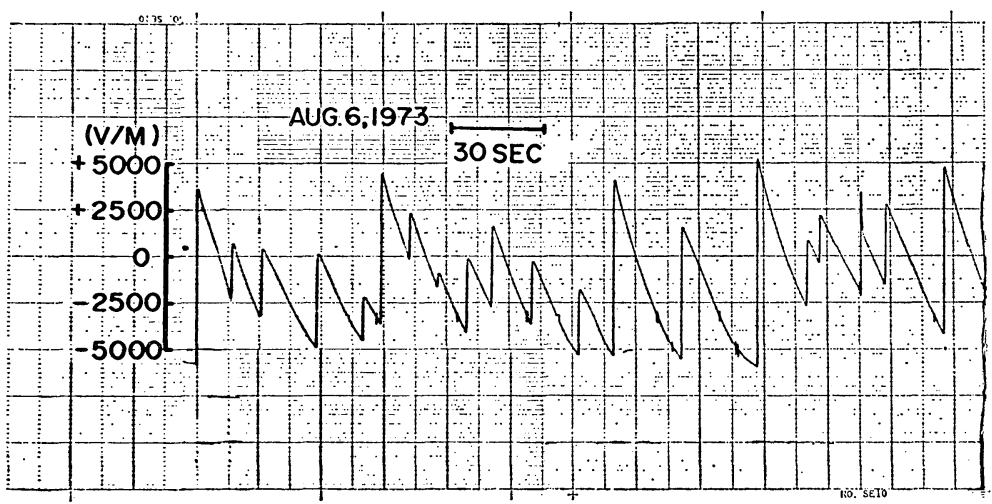

Fig. 2. Examples of field recoveries after close flashes. 


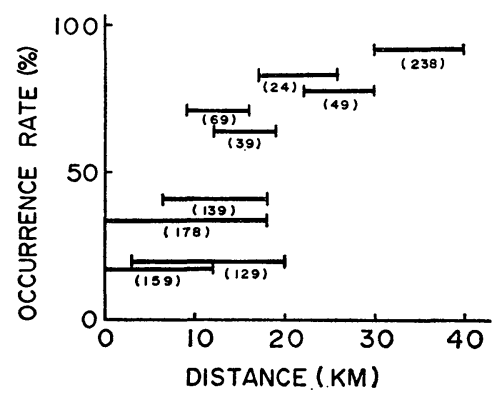

Fig. 3. Occurrence rate of exponential field recovery vs. distance. The numbers inside the parentheses show the numbers of flashes in each storm.

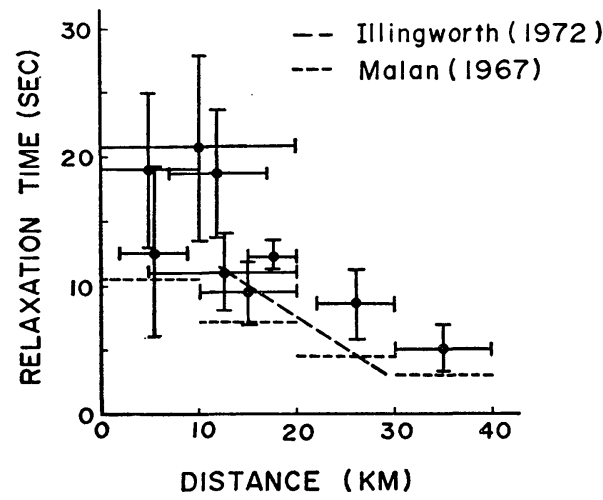

Fig. 4. Relaxation time of exponential recovery vs. distance. Relaxation times represent mean values within the distances shown by horizontal lines. - - - Illingworth (1972, Cambridge), ..... Malan (1967, Johannesburg).

$$
E(t)=A+B \exp (-t / \tau)
$$

where $\tau$ is the relaxation time, $A$ and $B$ are constants. The constants were determined by applying the least square method using the values of five sampled points on a recovery curve. In Fig. 4, mean values of relaxation times are shown against the distance. The results by Malan (1967) and Illingworth (1971) are also shown in the figure. Although present result shows the distance dependency similar to other results, the values we obtained are found to be almost 1.5 to 2 times larger than the values given by others.

Since the distance dependency of the relaxation times was obtained independently on the electrical activity of the storms, and it is unlikely that the average charge center heights change with storms, the field recovery for the distant flash almost determined by the redistribution of the space charges in the atmosphere (Illingworth, 1972). Therefore, the relaxation time for the distant flash is mainly effected by the spatial distribution of the electric conductivity in the atmosphere, which depends on the intensity of cosmic rays in the fair weather regions. Accordingly, it is concluded that the difference of the distance dependency in Fig. 4 is due to the difference of the distribution of the conductivity in the air surrounding thunderstorms.

\section{Close flash}

For close flashes, the occurence of exponential field recovery is limited to the case where flash intervals are very long. Excluding such a case, recovery curves are usually irregular, or nearly linear as shown in Fig. 2. Smith (1958) found that linear recoveries occurred when rainfall rate at the observation site exceeded about $75 \mathrm{~mm} / \mathrm{hr}$, and suggested that this was due to the splashing of raindrops near the ground. Fig. 5 shows the relationship between the rainfall rate at Imaiti and the types of field recoveries. Recovery types are not examined on the data on Aug. 3 because many of the recoveries that were recorded showed very small changes on the chart, but almost all displayed nearly linear recoveries. It is noticed that some of the flashes on Aug. 6 and Aug. 10 showed linear recoveries, though there was only little rainfall at the observation site. Therefore, it will be concluded, contrary to the result by Smith (1958), that the occurrence of linear recovery for a close flash seems to be independent on the rainfall rate at the observation site.

Figs. 6 and 7 show the time variations of the field recovery rates for linear recoveries, $\Delta E / \Delta t$, of the intensities of the field change due to flashes, $\Delta E$, and of the flash occurrence frequencies per minute, $N$. For the storm on Aug. 3 shown in Fig. 6, the development of $\Delta E / \Delta t$ and $N$ show the same trend, while $\Delta E$ does not vary with time on the average. Hence, the variation of $\Delta E / \Delta t$ is concluded to be caused not by the change in the distance to the flash, but by the change of the charge distribution in the clouds. For the storm on Aug. 6 shown in Fig. 7, $\Delta E$ decreases gradually with time after about $13 \mathrm{~h}$ $20 \mathrm{~m}$, while $N$ does not show such time variation. So the decrease of $\Delta E / \Delta t$ is thought to be caused 

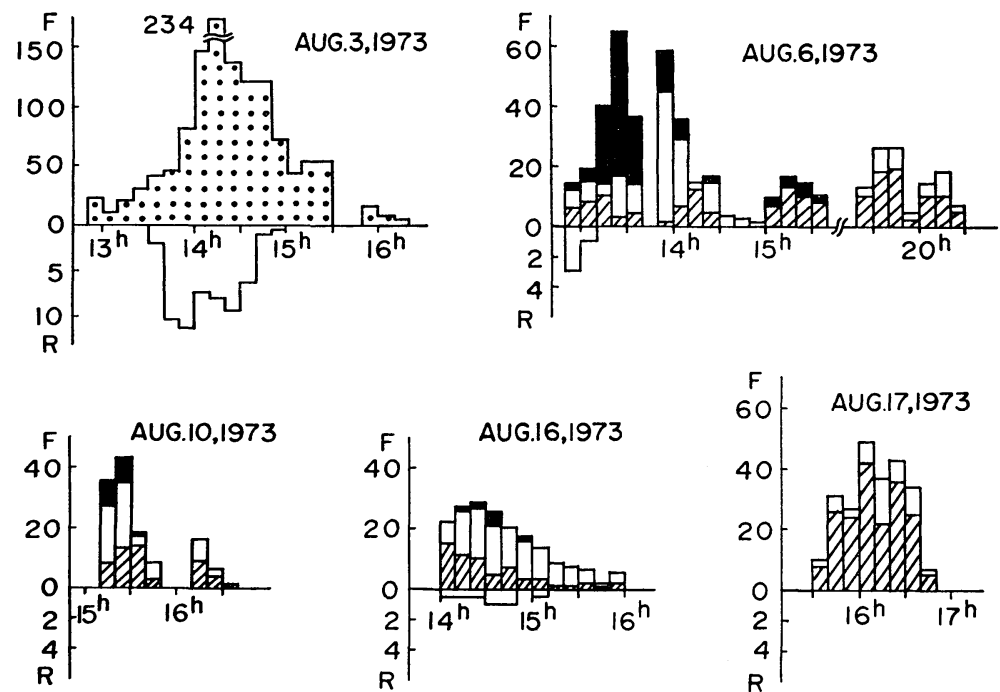

Fig. 5. Time variations of the flash frequencies, F, and, rainfall rate per 10 minutes, $\mathrm{R}$, of each storm. Shaded, hatched and brank portions represent the flash frequencies with approximately linear, exponential and complex recoveries respeciively. The types are not examined on the data on Aug. 3, 1973.
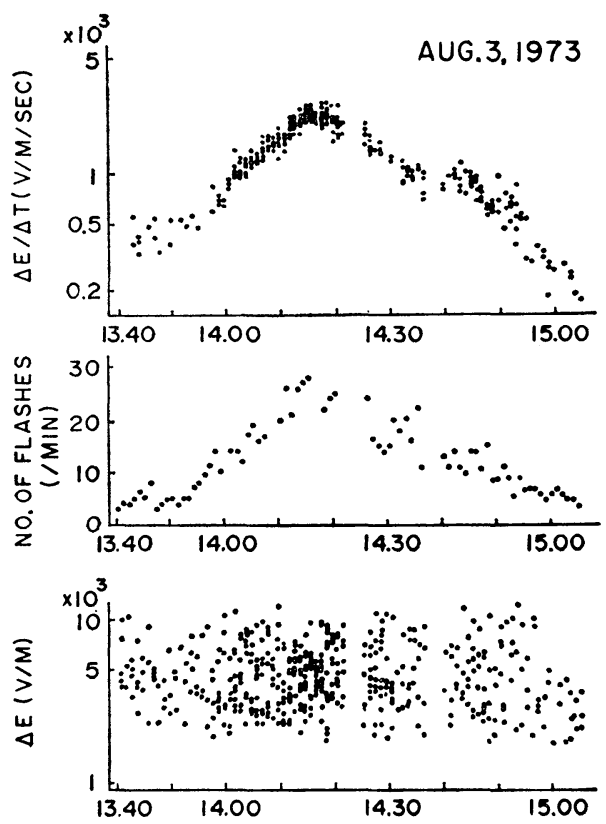

Fig. 6. Time variations of the field recovery rate, the flash occurrence frequencies per minute and the intensity of field change due to a flash.
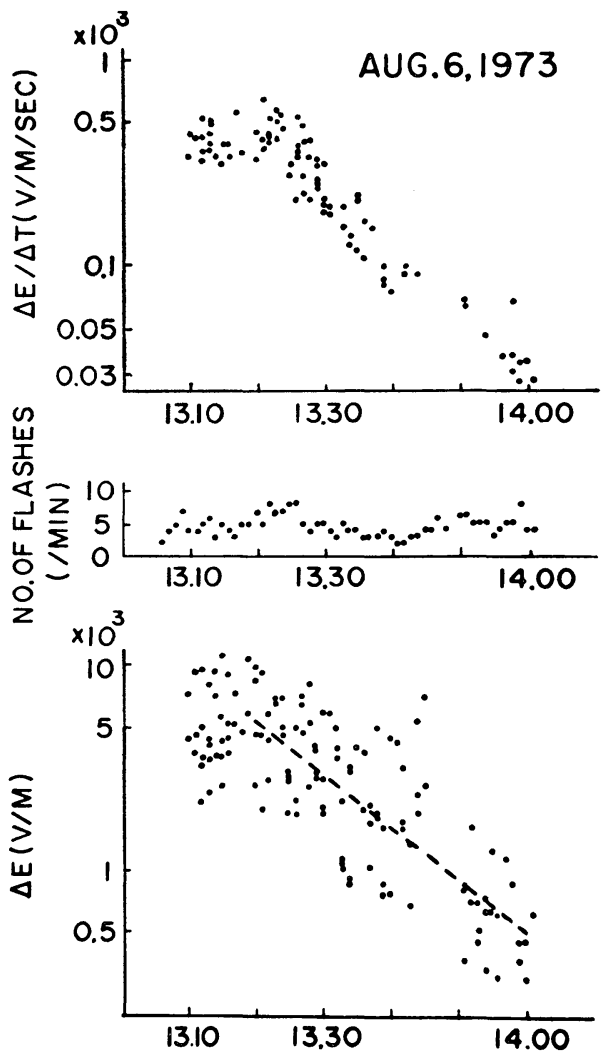

Fig. 7. Same as Fig. 6 except for Aug. 6. 
by the decrease in $\Delta E$ with increasing distance, which was verified through the movement of the radar echo. Accordingly, the change of $\Delta E$ caused by the change of the distance must be corrected in order to know the time variation of the charge distribution in clouds. Thus the correction was obtained by the following procedure. The change of $\Delta E$ was approximately represented by a broken line shown in Fig. 7, and it was assumed that $\Delta E$ would be kept constant at $6.3 \times 10^{3} \mathrm{~V} / \mathrm{m}$ after $13 \mathrm{~h} 20 \mathrm{~m}$, if the distance would have not changed. The corrected value of $\Delta E / \Delta t$ may be obtained by multiplying the value of $\Delta E / \Delta t$ by the ratio of $6.3 \times 10^{3} \mathrm{~V} / \mathrm{m}$ to the value on the broken line at the relevant time. In this way we obtained $\Delta E / \Delta t$ versus time curve representing time variation of charge distribution for the data on Aug. 6, and this time variation is shown in Fig. 8 .

The relationship between the field recovery rate and the occurrence frequency of flashes is shown

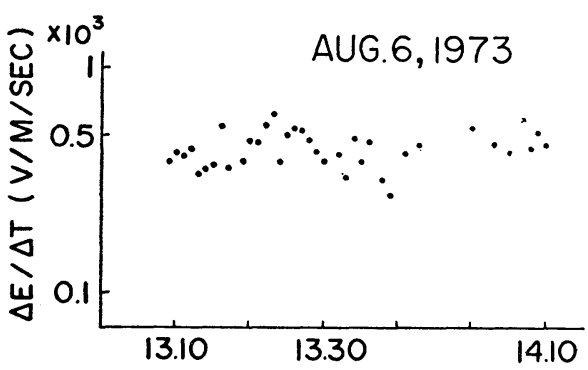

Fig. 8. Time variations of the field recovery rate for the storm on Aug. 6, 1973. The influence of the distance was corrected by the method given in text.

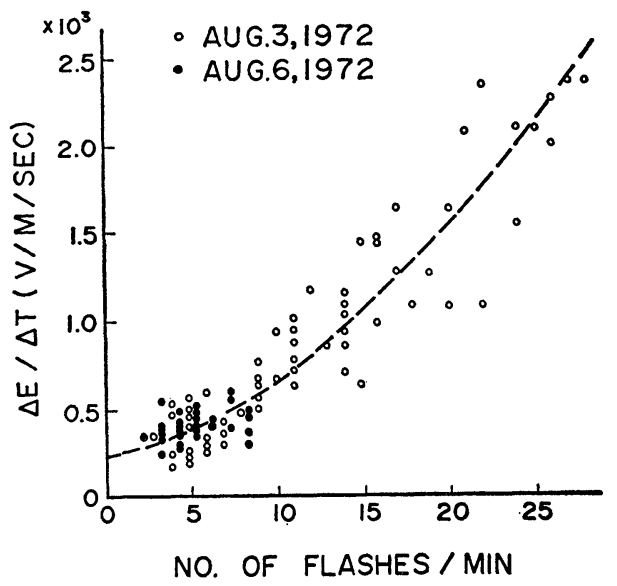

Fig. 9. Field recovery rate vs. number of flashes per minute. in Fig. 9. Closed circles and open circles correspond to the storm on Aug. 3 and Aug. 6 respectively, where for the data after $13 \mathrm{~h} 20 \mathrm{~m}$ on Aug. 6, the values shown in Fig. 8 were used. The broken line shows the best fit for all the plots, thus we obtain the following experimental expression,

$$
\frac{\Delta E}{\Delta t}=8.0 \times N^{1.7}+250
$$

where $\Delta E / \Delta t$ is in $\mathrm{V} / \mathrm{m} / \mathrm{sec}$ and $N$ is the occurrence frequency of flashes per minute. Adopting this, we can estimate $\Delta E / \Delta t$ even for distant flashes by counting $N$, provided $N$ is larger than about 3.

\section{Discussion}

For close flashes, the recovery curves are often regular and linear when the occurrence frequencies of flashes exceed about three per minute. A good correlation has been found between recovery rates and flash frequencies representing the electrical activity of the storm. This suggests the recovery rate to represent the rate of regeneration or of separation of charges in clouds.

Generally, the field change after a flash is produced by both the change of charge in the cloud and the space charges in the air surrounding the cloud. The steady state solution for the equation of the electric potential was given by Holzer and Saxon (1952) under the assumption of exponential increase with height of the conductivity,

$$
\phi=Q_{0} e^{-k\left(z-z_{\ominus}\right)} \frac{e^{-k R}}{R}+\text { image }
$$

where $\phi$ is the potential, $Q_{0}$ is the charge at the source $\left(Z=Z_{0}\right.$ and $\left.R=0\right), Z$ is the height, $R$ is the distance to the source and $k$ is the constant. We can calculate the potential due to space charges by subtracting the coulomb potential due to source charges from Eq. (3). Fig. 10 shows how the ratio of field intensities varies with the distance, assuming the heights of the sources at $3 \mathrm{~km}$ and $8 \mathrm{~km}$, and $k$ to be $1.1 \mathrm{~km}^{-1}$, where the ratio is seen to be almost less than unity for close distance. Therefore, the contribution of the space charges to the field change can be neglected for a close flash, and it may be said that the field recovery for a close flash represents mainly the time variation of the source charges, that is, 


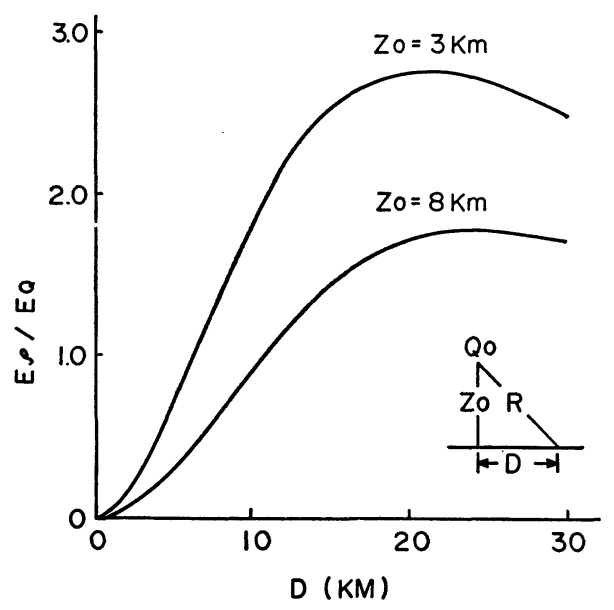

Fig. 10. Variation of $E_{\rho} / E_{Q}$ with distance. $E_{\rho}$ and $E_{Q}$ represent the field intensities due to space charge and the source charge respectively.

the regeneration or separation of charges.

Assuming a point charge at the source, the electric field on the ground is given by the following equation,

$$
E=A \frac{Q Z}{\left(D^{2}+Z^{2}\right)^{3 / 2}}
$$

where $Q$ is the charge, $Z$ is the height, $D$ is the horizontal distance and $A$ is the constant. Taking the time derivative keeping $D$ constant,

$$
\frac{d Q}{d t}=\frac{1}{A} \frac{\left(D^{2}+Z^{2}\right)^{3 / 2}}{Z} \frac{d E}{d t}+\frac{Q}{Z} \frac{2 Z^{2}-D^{2}}{D^{2}+Z^{2}} \frac{d Z}{d t}
$$

As $d E / d t$ is the order of $10^{3} \mathrm{~V} / \mathrm{m} / \mathrm{sec}$ from Figs. 6 and $7, d Z / d t$ represents vertical wind velocity and is less than about $30 \mathrm{~m} / \mathrm{sec}, Q$ is about 20 coulombs, $D$ and $Z$ are several kilometers, the second term of right-hand side in Eq. (5) can be neglected. This means that the electric field is not changed through the motion of the charges generated before a flash, as was suggested by Imyanitov and Shifrin (1962). Hence a field recovery rate is approximately proportional to the regeneration rate of the charge, and we obtain $d Q / d t$ is $1-10 \mathrm{coul} / \mathrm{sec}$ for the relevant values of $d E / d t, D$ and $Z$.

Since $d E / d t$ is constant with time for close flashes, $d Q / d t$ must be constant with time from Eq. (5), that is, $Q=c t$, which is the solution of the following equation, provided $\left(\lambda / \varepsilon_{0}\right) t \ll 1$,

$$
\frac{d Q}{d t}=c-\left(\frac{\lambda}{\varepsilon_{0}}\right) Q
$$

where $c$ is the constant which corresponds to the first term of right-hand side in Eq. (5), and $\lambda$ is the conductivity. Now $\lambda / \varepsilon_{0}$ being about 0.01 $\mathrm{sec}^{-1}$, the condition is satisfied within a time-length less than 10-20 seconds after a flash.

It is an important result for the charge generation in the cloud that the regeneration rate is $1-10 \mathrm{coul} / \mathrm{sec}$ and that this varies with time considerably, as is seen in Fig. 6.

From the above discussions, we can estimate quantitatively the regeneration rate of the charge for any distance from the information about the occurrence frequency of flashes. With respect to this point, Brook and Kitagawa (1960) reported that the occurrence frequency of flashes must be a measure of the rate of charge separation in isolated storm systems. On the theory of charge generation and separation, the relative velocity of the charge with respect to opposite sign is an important factor, which is usually replaced by the rainfall rate (Mason, 1971). Therefore it is very interesting to investigate the relationship between the occurrence frequency of flashes and the rainfall rate, as was pointed out by Brook and Kitagawa (1960). The relationship is now under investigation by the use of PPI radar data as a first step.

\section{Conclusion}

The dependency, on the flash distance, of the relaxation time of exponential recovery for the electric field after medium-distant and distant flash is found to be displayed differently from the results by other investigators.

For close flashes, a good correlation between the average recovery rate of the electric field and the occurrence frequency of flashes is found. It is concluded that the linear field recovery represents the regeneration of charge in thundercloud. The regeneration rate is estimated to be 1-10 $\mathrm{coul} / \mathrm{sec}$ from the data of the recovery rates. Thus, it is important to investigate how electrical activity depends on dynamical activity. One such approach may become possible by an examination of time variations of the occurrence frequency of flashes and the rainfall rate.

\section{Acknowledgements}

We would like to thank Prof. H. Ishikawa, Dr. T. Nakai and Dr. T. Takeuti of the Research Institute of Atmospherics, Nagoya University for their kind advices and discussions. We also wish to express our thanks to Dr. T. Ogawa of Kyoto 
University for his helpful and fruitful discussions. Thanks are due to Messrs. M. Nagatani and H. Nakada for their help in the instrumentation.

\section{References}

Brook, M. and N. Kitagawa, 1960: Some aspects of lightning activity and related meteorological conditions. J. Geophys. Res., 65, 1203-1210.

Illingworth, A. J., 1971: The variation of the electric field after lightning and the conductivity within thunderclouds. Quart. J.R. Met. Soc., 97, 440-456. , 1972: Electric field recovery after lightning as the response of the conducting atmosphere to a field change. ibid., 98, 604-616. Imyanitov, I. M. and K. S. Shifrin, 1962: Present state of research on atmospheric electricity. Soviet Physics Uspekhi, 5, 292-322.

Malan, D. J., 1967: The physics of the thunderstorm circuit. J. Franklin Inst., 283, 526-539.

Mason, B. J., 1972: The physics of the thunderstorms. Proc. R. Soc. London. A, 327, 433-466.

Michnowski, S., 1969: Some observations of electric field variations following lightning discharges. Planetary Electrodynamics, Gordon and Breach, 51-54.

Smith, L. G., 1958: Electric field studies of Frolida thunderstorms. Recent Advances in Atmospheric Electricity, Pergamon Press, 299-307.

Tamura, Y., 1954: An analysis of electric field after lightning discharges. J. Geomag. Geoelec., 6, 34-46.

\section{雷放電後の電場回復と雷雲内の電荷の再生成}

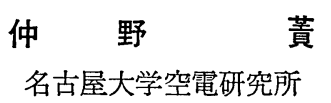

$10 \mathrm{~km}$ 以遠の雷放電の放電後の電場の指数関数型回復について，その緩和時間を調べた. 緩和時間は距雑ととも に減少するが，得られた值は他の研究者の值より 1.5〜2 倍大きい. 近距離の雷放電に関しては, 放電後の電場は遠 距離の場合と異って，注ぼ直線的に回復する。2 日間の雷雨について電場の回復率と放電頻度の間によい相関がある ことがわかった，回復率は雷雲内の電荷の再生成率と正相関があるので，距離と関係なく，放電頻度の観測から，雷 雲内の電荷の生成率を推定することが可能である。放電後の電荷の生成率は, この 2 日間の雷雨について, およそ 1〜10 クーロン/秒であることが見出された. 